\title{
Management of Fungal Rhinosinusitis - A Retrospective Study from a Tertiary Hospital in Kerala
}

\author{
Ike Thomas ${ }^{1}$, Tina Thomas², Reena Anie Jose ${ }^{3}$, Elizabeth Joseph ${ }^{4}$ \\ 1, 2, 3,4 Department of ENT, Believers Church Medical College Hospital, Tiruvalla, Kerala, India.
}

\section{ABSTRACT}

\section{BACKGROUND}

Fungal rhinosinusitis (FRS) is an increasingly common pathology in chronic rhinosinusitis and is often diagnosed late. The present study intended at analysing and understanding the clinical, pathological, microbiological, radiological characteristics of the disease in a tertiary hospital in Kerala.

\section{METHODS}

Case records of patients with pathologic or microbiologic diagnosis of FRS were retrospectively studied from January 2015 to January 2021, with reference to their demography, clinical presentation, comorbidities, and imaging features. The treatment aspects were also studied.

\section{RESULTS}

A total of 36 patients with pathological or microbiologic evidence of FRS were studied. There were $58 \%$ cases of allergic FRS, $33 \%$ cases of fungal ball and $8.3 \%$ cases of invasive FRS. $58 \%$ of patients were females, $77 \%$ patients had nasal block as their presenting symptom, and $66 \%$ of patients had duration of symptoms between 1-6 months. In radiological imaging, the maxillary sinus was most commonly involved. Treatment was always surgical removal. Allergic FRS (AFRS) needed prolonged topical steroids and invasive FRS needed systemic antifungals.

\section{CONCLUSIONS}

Our study suggests the importance of early diagnosis of FRS in all chronic rhinosinusitis patients by a high index of clinical suspicion. Tissue samples from the nose and sinuses should be studied for pathology and microbiology in all suspected cases to reach a diagnosis. Radiological imaging can aid in concluding diagnosis. Surgical options, supported by medical management play a vital role in the effective management of the disease.

\section{KEY WORDS}

Fungal rhinosinusitis, Allergic fungal rhinosinusitis, Fungal ball, Invasive fungal rhinosinusitis
Corresponding Author: Dr. Tina Thomas, M. S., Consultant ENT Surgeon \& Assistant Professor, Department of Otorhinolaryngology, Believers Church Medical College Hospital, Tiruvalla - 689 103, Kerala, India E-mail: tinaofficialent@gmail.com

DOI: $10.14260 /$ jemds/2021/685

How to Cite This Article:

Thomas I, Thomas T, Jose RA, et al. Management of fungal rhinosinusitis - a retrospective study from a tertiary hospital in Kerala. J Evolution Med Dent Sci 2021; 10(38):3374-3379, DOI: $10.14260 /$ jemds/2021/685

Submission 29-07-2021,

Peer Review 06-09-2021, Acceptance 13-09-2021, Published 20-09-2021.

Copyright (C) 2021 Ike Thomas et al. This is an open access article distributed under Creative Commons Attribution License [Attribution 4.0 International (CC BY 4.0)] 


\section{BACKGROUND}

Fungi are increasingly being recognised as a cause of chronic rhinosinusitis (CRS). Being ubiquitous in nature, fungal spores are continuously being inhaled and stored in the respiratory mucosa. Healthy individuals have mostly saprophytic responses but fungi cause disease in certain conditions related to hosting defence. ${ }^{1}$ Classification of fungal rhinosinusitis (FRS) has been controversial. The consensus is that they are of two types, invasive and non-invasive. The invasive FRS is thought to be an infective process, whereas it is not clear whether the inflammation in the non-invasive FRS is caused by fungal colonisation or acute infection. ${ }^{2}$ Allergic fungal rhinosinusitis (AFRS) was defined as a subset of FRS characterised by Type I allergic reaction to fungi, whereas some studies reported sensitisation to fungi in virtually all cases of CRS when IgE mediated allergy was not observed, thus suggesting 'eosinophilic FRS' as a term for AFRS. ${ }^{3}$ FRS is now understood not to be a virtually continuous spectrum of pathology but the disease states are distinct and involve discrete pathologic diagnoses that rarely show a transition from one condition to another. ${ }^{4}$ Irrespective of subtype, the diagnosis of FRS is by proving the presence of fungus in the tissue by pathology or microbiology. The diagnosis of FRS is made with clinical suspicion, radiological imaging and proven by microbiological / histopathologic presence of fungus. Treatment is by surgical removal of fungus and inflammatory tissue with systemic antifungal when indicated.

The pathophysiology of the host and environmental factors in the disease progression is not fully understood though the diagnostic methods have improved. ${ }^{5}$ The increasing use of broad-spectrum antibiotics, immunosuppressive therapy, immunodeficiency states, cancer chemotherapy and increased use of intensive care interventions are thought to be causative factors. When the presence of fungus within the nose and paranasal sinus starts making pathological changes, the symptoms of nasal obstruction and nasal discharge set in. Patients tend to ignore the symptoms or may find temporary relief with local medical treatment. Polypoidal changes within the nose are treated symptomatically by otolaryngologists with antibiotics, steroids and nasal sprays. The pressure symptoms of FRS on the eye causing eye pain, watering and swelling around the eyes are treated by ophthalmologists with broad-spectrum antibiotics, steroids and antibiotic eye drops. The headache and facial pain get treated by physicians and neurologists with antibiotics and migraine prophylactics. When the symptoms persist and cause a significant effect on their daily life, they seek detailed evaluation. Many of them present to the Department of Otorhinolaryngology with severe morbidities of eye pain, swelling and watering of eyes, severe facial pain and headache, often at the end of several months of the onset of less intense symptoms. Early identification of diagnosis with clinical suspicion and the use of diagnostic methods to initiate prompt treatment would help to bring down the burden of disease. With the increasing number of cases of fungal sinusitis in our CRS patients, this study aimed at understanding FRS in our geographical location.

\section{Objectives}

1. To study the demographic, clinical and radiological features across the subsets of FRS.

2. To study the pathological and microbiological features of the disease subtypes.

3. To study the treatment and recurrence pattern of the disease.

\section{METHODS}

This was a retrospective review of 36 cases of FRS that were diagnosed at the Department of Otorhinolaryngology, Believers Church Medical College Hospital, Tiruvalla, Kerala, India. This was a descriptive study in design. Ethics committee approval was obtained from the institutional research committee. Data on patients who underwent nasal surgery were collected from January 2015 to January 2021, from the hospital records. Patients diagnosed with fungal sinusitis from the surgical and pathologic findings were considered. Inclusion criteria were cases with histopathology or microbiology proved FRS. The study excluded patients with other types of chronic sinusitis and sinonasal polyposis. The following data were collected:

1. Demographic data - age and gender

2. Presence of comorbidities (Immunocompromised conditions)

3. Clinical picture - presenting symptoms, duration since onset

4. Imaging findings- side involved (right, left, or bilateral), sinus involved (sphenoid, maxillary, frontal, and/or ethmoid), presence of mucosal thickening, complete opacification, increased intrasinus attenuation, sinus expansion, remodelling / wall thinning and the involvement of adjacent soft tissues in each involved sinus

5. Laboratory data - blood eosinophil rate

6. Pathology findings - the presence of eosinophilic mucin, eosinophils, fungal hyphae, and Charcot-Leyden crystals

7. Microbiology findings, $\mathrm{KOH}$ mount and fungal culture

8. Management, including therapeutic strategy and postoperative medication (antihistaminic agents, antifungal drops, corticosteroids, and antibiotics).

\section{Statistical Analyses}

Statistical analyses of the data were performed. The prevalence of AFS was calculated as the percentage of patients diagnosed and treated as AFS among all cases of chronic rhinosinusitis was surgically treated in the same period. Descriptive statistical tools (Frequency and percentage) were used to describe the demographic and clinical characteristics, as well as the imaging and pathological characteristics.

\section{RESULTS}

Altogether there were 172 cases of functional endoscopic sinus surgeries in our hospital from January 2015 to January 
2021. We had 36 cases of fungal rhinosinusitis diagnosed by histopathology or microbiology, which corresponded to 20.9 $\%$ of our total cohort of chronic sinusitis cases. Of the total patients, 3 ( $8.3 \%$ ) were invasive FRS, where tissue invasion was seen in addition to fungal elements on histopathology. 21 patients (58.3\%) belonged to AFRS, based on the presence of allergic mucin and fungal elements on histopathology with supporting radiologic findings. 12 (33.3 \%) cases were a fungal ball, based on fungal elements without allergic mucin, with characteristic radiologic findings.

There were 21 female patients (58.3\%) and 15 (41.7\%) male patients. Our youngest patient was 11 years old and the eldest patient was 74 years, mean age of the patients was 47.9 years. The $21-40$ age group and the above 60 age groups were mostly affected [Table 1].

\section{Clinical Findings}

Most of our FRS patients presented with a nasal block (75 \%); the other symptoms were facial pain with headache, nasal discharge, blood-stained discharge, eye pain with swelling, swelling of face localised to sinus locations. Out of 27 patients with nasal block, most of them had AFRS (70.3 \%). Localised facial pain and headache were seen in $75 \%$ of patients with the fungal ball. The least common presentation was bloodstained nasal discharge, out of which AFRS was the most common entity. Duration of symptoms ranged from 5 days to many years, mean duration was 3.22 months. History of previous nasal surgery was present in $16.6 \%$ of cases, 3 in the invasive group and 3 in the AFRS group. Among the comorbidities, diabetes was the most common entity, and it was common in the fungal ball group. Peripheral eosinophilia was observed in 4,100\% cases in the AFRS group and absent in other types.

\section{Radiologic Findings}

Deviated nasal septum (DNS) was observed in $78 \%$ of cases. Bilateral involvement was seen in $25 \%$ of AFRS cases. All cases of invasive FRS were unilateral and involved maxillary, ethmoid and frontal sinuses on the affected side. The most commonly involved sinus was the maxillary sinus in all types of fungal sinusitis. Maxillary (95.2\%) and ethmoid (90.4\%) sinuses were affected in AFRS; maxillary sinuses were affected in $91.6 \%$ of cases of the fungal ball. Intrasinus attenuation and mucosal opacification were the most consistent radiological findings on CT in $83.3 \%$ and $86.11 \%$ cases respectively. Remodelling and thickening of the walls of the sinus were seen in $66.6 \%$ and $71.4 \%$ cases of AFRS. Soft tissue involvement was seen in $66.6 \%$ of cases of invasive FRS and $28.5 \%$ cases of AFRS [Table 1].

\section{Histopathology and Microbiology Findings}

Histopathology studies of the 29 cases were available, which showed respiratory epithelium with mixed inflammatory cell infiltrate in all the cases. Allergic mucin was seen in 14 cases of AFRS and 1 case of the fungal ball (Figure 1). Fungal hyphae (thin septate filaments) were demonstrated in 15 cases of AFRS and 7 cases of the fungal ball. In cases of invasive fungal sinusitis, intense inflammatory reaction in mucosa with extensive necrosis was observed. Fungal elements (broad aseptate hyphae) and angioinvasion were evident as well, suggestive of mucormycosis. On microbiology analysis, fungal elements were demonstrated in 23 cases (63.8\%)(Figure 2). Out of 10 samples sent for fungal culture, 4 cases proved fungal growth, all of which showed Aspergillus flavus (40\%) (Figure 3,4).

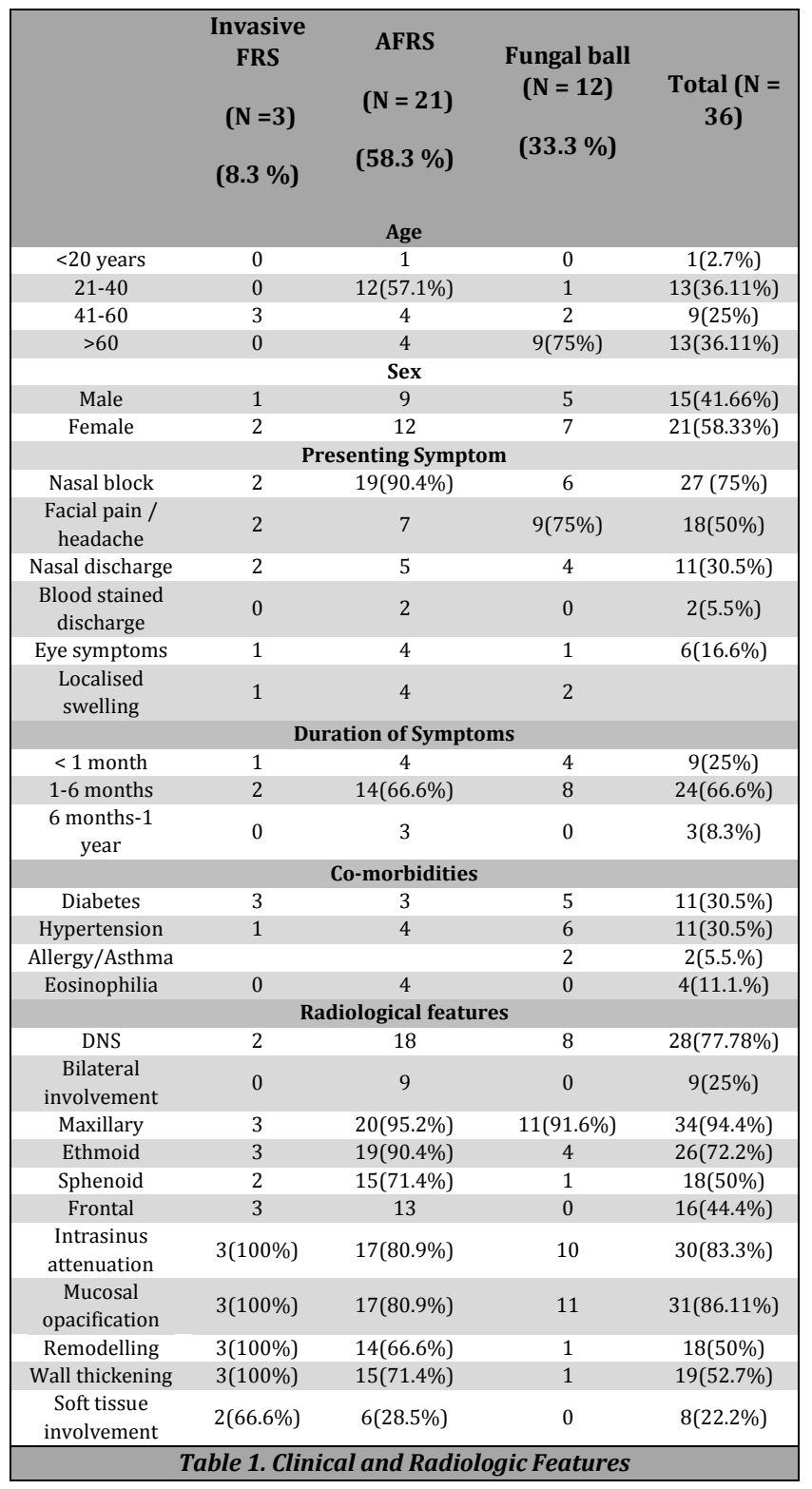

\section{Management and Post-Operative Follow-Up}

All patients received a perioperative short course of antibiotics, analgesics and were started on saline nasal douches on the second postoperative day. Surgery was done for disease clearance and ventilation of the sinuses. Postoperative follow-ups were done in a 10-day interval, where nasal endoscopic cleaning was performed. Steroid nasal douches were started for AFRS patients on their first follow up visit. 14 cases of AFRS were relieved of their symptoms after surgery, 5 were lost after 3-4 follow up visits and 2 had a recurrence of nasal polyps. 10 cases of fungal balls were completely relieved, 2 were lost after 3-4 followup visits. 1 case of invasive fungal sinusitis underwent revision surgery and 2 patients received antifungal drugs (IV amphotericin, oral posaconazole) and were on follow up for a 
year. 1 patient with invasive FRS died 12 days after the surgery due to other comorbidities.
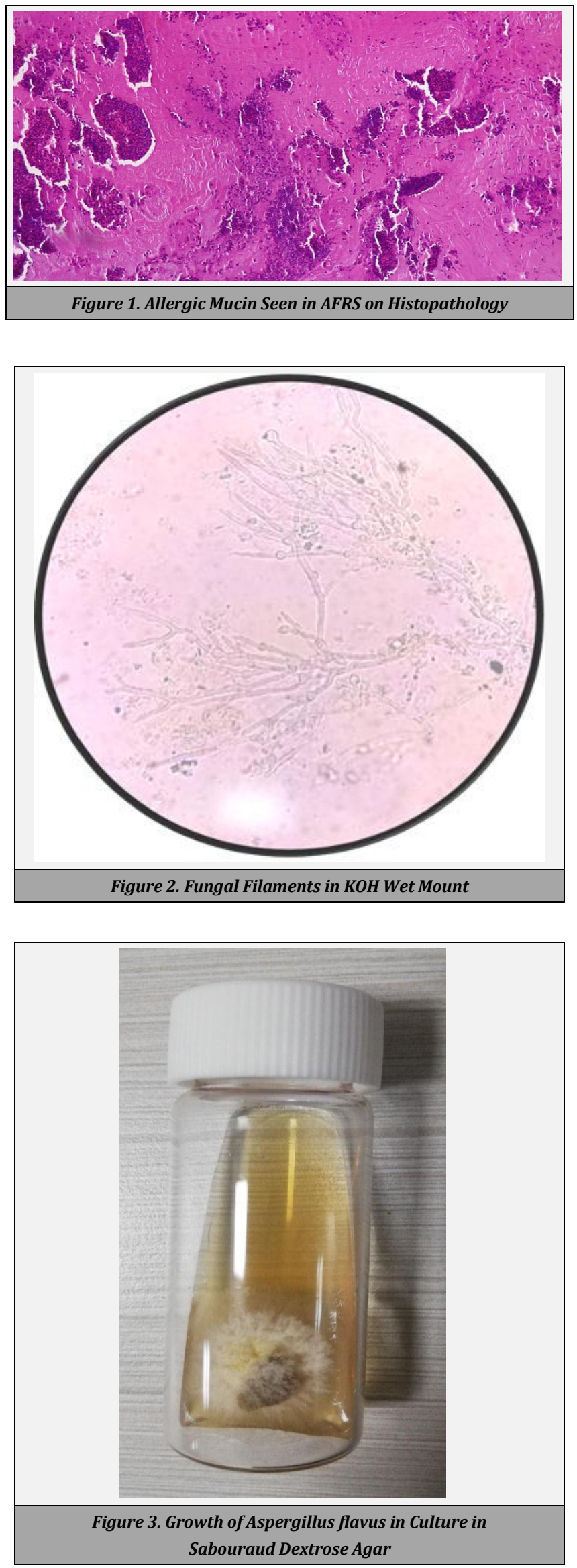

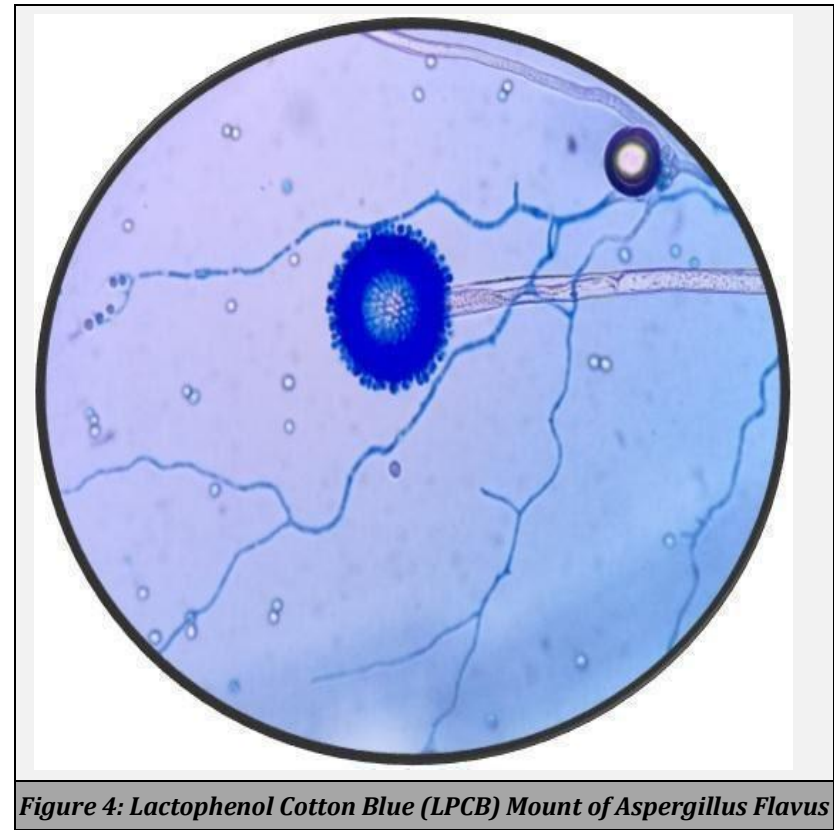

\section{DISCUSSION}

In our patient group of surgical CRS patients, $21 \%$ (36/172) were observed to have FRS. The incidence of FRS reported in our patient group was very well comparable to that reported in the literature. In a recent study from Kerala, there were $26.9 \%$ of FRS cases. ${ }^{6}$ In another study from Karnataka, $26 \%$ prevalence of fungal sinusitis by fungal culture among CRS patients were observed. ${ }^{7}$ Das et al. at Chandigarh, reported fungal rhinosinusitis with an incidence of $42.7 \%$ of all the 665 cases of chronic rhinosinusitis over 5 years. ${ }^{8}$ In a further retrospective study on surgical samples of chronic sinusitis in Singapore*, $8.3 \%$ of chronic sinusitis were fungal in origin. ${ }^{5}$ The prevalence of the disease in our study in Kerala, having hot and humid tropical climate and the rich vegetation is selfexplanatory.

Most of our patients had allergic FRS (58.3\%), followed by the fungal ball (33.3 \%) and invasive FRS ( $8.3 \%$ ) was least common, which compares to other studies reported in the literature. In a study from South India, where FRS was diagnosed by microbiology analysis of 211 samples, $63 \%$ were AFRS, $34 \%$ were invasive and $3 \%$ were fungal granulomas. ${ }^{9}$ In the present study, Aspergillus flavus was the commonest isolate which was comparable with other studies across the country $(6,7,9,10)$. Other than aspergillus spp., dematiaceous fungi were the commonest reported isolates in AFRS. ${ }^{9,10}$

The classification of FRS has always been a cause for confusion. Bent and Kuhn criteria for AFRS and De Shazo criteria for invasive FRS and fungal balls are widely used to arrive at a diagnosis. ${ }^{11,12}$ Though pathological evidence of fungus is diagnostic, clinical and radiologic features are considered as valid criteria for diagnosis. In our study, we considered histopathologic or microbiological proof of fungus at the beginning of the study. Adapting the laid down criteria, samples with tissue invasion were categorised as invasive FRS, samples with the presence of allergic mucin and fungal hyphae and supporting radiological findings were categorised as AFRS, dense fungal hyphae without allergic mucin with its 
radiologic findings were included as fungal ball. There were no cases of chronic invasive or granulomatous changes in our pathology studies. AFRS is the most common type of FRS in our study, as in previous studies from India. ${ }^{13,14}$

Most of our patients were females. The age group of 2140 and above 60 groups were most affected. In a study from Andhra Pradesh, $24.5 \%$ of fungal sinusitis cases belonged to the 20 - 29 years age group, ${ }^{15}$ while in a study from Kerala, 36 - 55 years age group were mostly affected. ${ }^{16} 21$ - 40 years were most affected in another study reported from Karnataka. $^{7} 57 \%$ of our AFRS patients were in the 21- 40 years age group while $75 \%$ of fungal ball patients were in the above 60 age group. AFRS is a disease of adolescents and young adults, ${ }^{17}$ where males predominate in children and females among adults. In our study, young female adults were more affected. Most of the patients had nasal block as their presenting symptom. The nasal block was the predominant clinical symptom in similar studies.7,15 Most of our patients had the persistent nasal block, which was not responding to conventional medical management of CRS. This triggers the physician to evaluate for a fungal element in the pathology of symptoms. Peripheral eosinophilia was noted in $11 \%$ and all of them belonged to the AFRS group. AFRS is understood to be a type I allergic reaction to the presence of fungus in the sinus lumen, where the tissue oedema due to inflammation results in polypoidal changes blocking sinus ostia and CRS.

The most commonly involved sinus on CT imaging in our studies was the maxillary sinus in all types of fungal sinusitis. Intrasinus attenuation and mucosal opacification were the most consistent radiological findings on CT in $83.3 \%$ and $86.11 \%$ cases respectively. Remodelling and thickening of the walls of the sinus were seen in $66.6 \%$ and $71.4 \%$ cases of AFRS. Soft tissue involvement was seen in $66.6 \%$ cases of invasive FRS and $28.5 \%$ cases of AFRS. Maxillary and ethmoids were mostly affected for AFRS in our study. In AFRS, there was often pansinusitis or bilateral involvement of multiple sinuses, with ethmoid involvement being the most common. ${ }^{17}$ There was hyperattenuating and opacification of sinus lumen due to allergic mucin while mucosal linings appeared hypointense, with expansion and remodelling of bony sinus walls. ${ }^{18} 91.6 \%$ cases of fungal ball involved maxillary sinus. Classical findings in CT demonstrated the involvement of only one paranasal sinus with a hyperintense ("metal-dense") spot at the centre of the fungus ball, often with sclerosis of the adjacent bone, taking contour of the sinus lumen. ${ }^{18}$ Our CT findings were consistent with other reports existing in the literature.

Histopathology of fungal sinusitis in our study showed allergic mucin and fungal hyphae in $51.7 \%$ and $75.8 \%$ cases. Bharadwaj et al. in their study reported the presence of allergic mucin in $38 \%$ of cases who underwent surgery. ${ }^{19}$ Das et al. reported in their histopathology study $56.3 \%$ AFRS, 3.9 $\%$ fungal ball, $16.9 \%$ chronic granulomatous FRS, $1.4 \%$ chronic invasive FRS, $17.2 \%$ acute fulminant FRS and also $4.25 \%$ mixed histopathological patterns. ${ }^{8}$ Chronic granulomatous or chronic invasive FRS were not observed in our patient cohort.

FRS is a surgically treated disease and there was a relief of symptoms in $69.4 \%$ of cases. Saline nasal douches were part of routine post-surgical care. Steroid douches were started in all AFRS cases as the disease is primarily an allergic reaction of the tissue to the presence of fungus. It is needed for prolonged durations in AFRS patients as fungi are always present in their environment and patients are sensitized already. Recurrence of symptoms is known and can be managed medically with steroid douches and endoscopic cleaning. Revision surgery may also be needed. The fungal ball gets treated completely with clearance of the dense fungus mass from the nose and sinus. Invasive FRS often needs repeated surgeries for debridement of necrotic tissue, along with systemic antifungals. In a study from Singapore, there was $13.6 \%$ recurrence when followed up to 60 months. ${ }^{5}$

\section{CONCLUSIONS}

There is an increasingly high incidence of FRS among CRS patients. AFRS was common among the subtypes. Nasal block was the commonest presentation. This is always a surgically treated disease. The identification of the presence of fungus by histopathology or microbiology is always indicated and mandatory to categorise the disease. Samples should be sent from all suspected cases. Radiological imaging can aid in concluding diagnosis. AFRS needs surgical clearance and a prolonged post-op topical steroid, recurrence was seen. The fungal ball is completely treated by surgical removal of the fungus while invasive FRS needs surgical debridement and systemic antifungals.

\section{Limitations of the Study}

This article reports our experience of management of FRS and our study has several limitations. The data is from a single centre and the sample size is rather small and the study design is retrospective. We were not able to determine the presence of atopy, which is one of the crucial criteria for the diagnosis of AFRS. Laboratory investigations were not routinely ordered for all patients, there was a lack of data in all the parameters. Clinicians were probably not aware of the importance of sending tissue samples for pathology and microbiology analysis, not to miss a diagnosis. Despite all these limitations, we strongly believe that the analysis of the data helped us to understand the pathology involved and its incidence and diagnosis better and manage these groups of patients effectively.

Data sharing statement provided by the authors is available with the full text of this article at jemds.com.

Financial or other competing interests: None.

Disclosure forms provided by the authors are available with the full text of this article at jemds.com.

\section{REFERENCES}

[1] Patrascu E, Manea C, Sarafoleanu C. Difficulties in the diagnosis of fungal rhinosinusitis - literature review. Romanian Journal of Rhinology 2016;6(21):11-17.

[2] Meltzer EO, Hamilos DL, Hadley JA, et al. Rhinosinusitis: establishing definitions for clinical research and patient care. J Allergy Clin Immunol 2004;114(6 Suppl):155-212. 
[3] Ponikau JU, Sherris DA, Kita H, et al. Intranasal antifungal treatment in 51 patients with chronic rhinosinusitis. J Allergy Clin Immunol 2002;110(6):862-6.

[4] Adelson RT, Marple BF. Fungal rhinosinusitis: state-ofthe-art diagnosis and treatment. J Otolaryngol 2005;(34 Suppl 1):S18-23.

[5] Alshaikh NA, Alshiha KS, Yeak S, et al. Fungal rhinosinusitis: prevalence and spectrum in Singapore. Cureus 2020;12(4):e7587.

[6] Archana, Sindhu VN, Salima RW. Chronic rhinosinusitisfungal etiology \& clinical, histopathological, radiological profile in a tertiary care centre. Journal of Medical Science and Clinical Research 2018;6(9):863-8.

[7] Syiemlieh B, Mariraj J. Study on chronic rhinosinusitis: a clinico-mycological perspective in a tertiary care centre. International Journal of Current Microbiology and Applied Sciences 2020;9(7):3740-7.

[8] Das A, Bal A, Chakrabarti A, et al. Spectrum of fungal rhinosinusitis: Histopathologist's perspective. Histopathology 2009;54(7):854-9.

[9] Michael RC, Michael JS, Ashbee RH, et al. Mycological profile of fungal sinusitis: an audit of specimens over a 7year period in a tertiary care hospital in Tamil Nadu. Indian Journal of Pathology and Microbiology 2008;51(4):493-6.

[10] Kaur R, Lavanya S, Khurana N, et al. Allergic fungal rhinosinusitis: a study in a tertiary care hospital in India. Journal of Allergy 2016;2016:7698173.
[11] Bent JP 3rd, Kuhn FA. Diagnosis of allergic fungal sinusitis. Otolaryngol Head Neck Surg 1994;111(5):5808.

[12] deShazo RD, O'Brien $M$, Chapin $K$, et al. A new classification and diagnostic criteria for invasive fungal sinusitis. Arch Otolaryngol Head Neck Surg 1997;123(11):1181-8.

[13] Munjal M, Gupta V, Chinna D, et al. Demographic profile of fungal rhinosinusitis at a tertiary care centre. Int J Otorhinolaryngol Head Neck Surg 2021;7(1):61-5.

[14] Chakrabarti A, Rudramurthy SM, Panda N, et al. Epidemiology of chronic fungal rhinosinusitis in rural India. Mycoses 2015;58(5):294-302.

[15] Waghray J. Clinical study of fungal sinusitis. Int J Otorhinolaryngol Head Neck Surg 2018;4(5):1307-12.

[16] Santhi T, Rajan KV. Presence of fungal organisms in chronic rhinosinusitis with nasal polyposis: a clinico pathological study from Kerala. Int J Sci Stud 2015;3(3):99-104

[17] Al-Dousary SH. Allergic fungal sinusitis: radiological and microbiological features of 59 cases. Ann Saudi Med 2008;28(1):17-21.

[18] Pillai S, Bhandarkar AM, Nair SS, et al. A radiological profile of fungal sinusitis. Asian Journal of Pharmaceutical and Clinical Research 2017;10(9):331-4.

[19] Bhardwaj BB, Gill JS, Tanya S. The role of CT scan and histopathology in diagnosis of allergic fungal sinusitis. Clinical Rhinology an International Journal 2014;7(3):117-20. 